\title{
openheart COVID-19 pandemic and STEMI: pathway activation and outcomes from the pan-London heart attack group
}

Callum D Little (10 , ${ }^{1,2}$ Tushar Kotecha, ${ }^{1}$ Luciano Candilio, ${ }^{1}$ Richard J Jabbour, ${ }^{3}$ George B Collins, ${ }^{4}$ Asrar Ahmed, ${ }^{5}$ Michelle Connolly, ${ }^{6}$ Ritesh Kanyal (D) ,7 Ozan M Demir, ${ }^{8}$ Lucy O Lawson, ${ }^{1}$ Brian Wang, ${ }^{3}$ Sam Firoozi, ${ }^{6}$ James C Spratt, ${ }^{6}$ Divaka Perera, ${ }^{8}$ Philip MacCarthy (D) , ${ }^{7}$ Miles Dalby, ${ }^{5}$ Ajay Jain, ${ }^{4}$ Simon J Wilson, ${ }^{6}$ lqbal Malik, ${ }^{3}$ Roby Rakhit ${ }^{1,2}$

\begin{abstract}
- Additional material is published online only. To view, please visit the journal online (http://dx.doi.org/10.1136/ openhrt-2020-001432).

To cite: Little CD, Kotecha T, Candilio L, et al. COVID-19 pandemic and STEMl: pathway activation and outcomes from the pan-London heart attack group. Open Heart 2020;7:e001432. doi:10.1136/ openhrt-2020-001432
\end{abstract}

Received 28 August 2020 Revised 11 September 2020 Accepted 21 September 2020

D Check for updates

(c) Author(s) (or their employer(s)) 2020. Re-use permitted under CC BY. Published by BMJ.

For numbered affiliations see end of article.

Correspondence to

Dr Callum D Little; callumlittle@ nhs.net

\section{ABSTRACT}

Objectives To understand the impact of COVID-19 on delivery and outcomes of primary percutaneous coronary intervention (PPCl). Furthermore, to compare clinical presentation and outcomes of patients with ST-segment elevation myocardial infarction (STEMI) with active COVID-19 against those without COVID-19.

Methods We systematically analysed 348 STEMI cases presenting to the PPCl programme in London during the peak of the pandemic (1 March to 30 April 2020) and compared with 440 cases from the same period in 2019. Outcomes of interest included ambulance response times, timeliness of revascularisation, angiographic and procedural characteristics, and in-hospital clinical outcomes

Results There was a $21 \%$ reduction in STEMI admissions and longer ambulance response times (87 (62-118) min in 2020 vs $75(57-95) \min$ in $2019, p<0.001)$, but that this was not associated with a delays in achieving revascularisation once in hospital (48 (34-65) min in 2020 vs $48(35-70)$ min in $2019, p=0.35$ ) or increased mortality ( $10.9 \%$ (38) in 2020 vs $8.6 \%$ (38) in 2019, $p=0.28$ ). 46 patients with active COVID-19 were more thrombotic and more likely to have intensive care unit admissions $(32.6 \%$ (15) vs $9.3 \%$ (28), 0R $5.74(95 \% \mathrm{Cl} 2.24$ to 9.89$), \mathrm{p}<0.001)$. They also had increased length of stay (4 (3-9) days vs 3 $(2-4)$ days, $p<0.001)$ and a higher mortality $(21.7 \%$ (10) vs $9.3 \%$ (28), OR 2.72 (95\% Cl 1.25 to 5.82 ), $p=0.012$ ) compared with patients having PPCI without COVID-19. Conclusion These findings suggest that PPCI pathways can be maintained during unprecedented healthcare emergencies but confirms the high mortality of STEMI in the context of concomitant COVID-19 infection characterised by a heightened state of thrombogenicity.

\section{INTRODUCTION}

The current COVID-19 global pandemic has required urgent restructuring of established clinical pathways in order to manage the surge of patients presenting with SARS-CoV-2. As of 17 July 2020, there have been more than 13 million confirmed cases of COVID-19 worldwide and 584940 deaths. $^{1}$ In the UK,

\section{Key questions}

What is already known about this subject?

- The rates of ST-segment elevation myocardial infarction (STEMI) admissions worldwide have decreased during the COVID-19 pandemic.

- Single-centre data suggest disruption to emergency services may result in delays to the primary percutaneous coronary intervention (PPCl) pathway including time from pain onset to first medical contact and in-hospital delivery of revascularisation.

- The systemic inflammatory response, induced by COVID-19, appears to disrupt antithrombotic mechanisms contributing to a higher incidence of thrombotic complications. The implications for acute coronary syndromes remain unclear.

What does this study add?

- Consistent with international findings there has been a $21 \%$ decrease in STEMI cases in London during the COVID-19 pandemic compared with the same period in 2019.

- Modifications to the existing in-hospital PPCl pathways, such as routine use of personal protective equipment and redeployment of staff to other clinical areas, do not result in delayed time taken to achieve coronary revascularisation (door to balloon time) or worse clinical outcomes

- Ambulance response times have increased during the pandemic period, likely due to the high volume of COVID-19-illness related calls.

- Patient with COVID-19 and STEMI have a significantly higher coronary thrombus burden compared with COVID-19 negative patients.

daily admissions to hospital with COVID-19 peaked at 3260 on 1 April $2020 .^{2}$

Additionally, COVID-19 poses a risk for healthcare workers, necessitating routine use of personal protective equipment (PPE) and modifications to existing medical services and pathways. ${ }^{3}$ In particular, managing patients with suspected or proven 


\section{Key questions}

How might this impact on clinical practice?

- A targeted antithrombotic approach may be of benefit in these patients but further prospective studies are required.

- Systemic thrombolysis has been suggested as an alternative strategy to managing patients with STEMI during the pandemic; however, our data suggest that even at the peak of COVID-19 related admissions to hospital, it has been possible to maintain effective $\mathrm{PPCl}$ services

COVID-19 represents a significant risk to the delivery of a primary percutaneous coronary intervention (PPCI) pathway where time-dependent revascularisation is key to successful outcomes. ${ }^{4}$ It has been suggested that the current pandemic may result in delays to the pathway including time from pain onset to first medical contact and in-hospital delivery of revascularisation. ${ }^{3}$

Moreover, COVID-19 may have a significant impact on presentation, angiographic findings ${ }^{56}$ and clinical outcomes of patients presenting with ST-segment elevation myocardial infarction (STEMI) through the PPCI pathway. ${ }^{7}$ The systemic inflammatory response, induced by COVID-19, appears to disrupt antithrombotic mechanisms. ${ }^{8}$ Furthermore, cellular viral inclusions and the resultant inflammation may produce endothelial injury, ${ }^{9}$ thrombotic microangiopathy ${ }^{10}$ and microvascular dysfunction further potentiating a procoagulopathic state and contributing to the higher incidence of thrombotic complications identified in these patients. ${ }^{11}$

\section{AIMS}

To evaluate the effect of the COVID-19 pandemic on an established ambulance-triggered PPCI programme involving seven high-volume heart attack centres in London, UK. Data and outcomes from 2020 were compared with the same time period from 2019. Furthermore, we sought to compare clinical presentation and outcomes of patients with STEMI with active COVID-19 against those of patients with STEMI without COVID-19.

\section{METHODS}

\section{Study design and patient population}

The PPCI programme in London is the largest urban network of seven heart attack centres in the UK using a single ambulance triggered service and providing $24 / 7$ treatment for STEMI to a population of 9 million. We conducted a retrospective observational analysis of consecutive PPCI pathway activations to all seven heart attack centres in London, UK. The study period was 1 March to 30 April 2020, corresponding with the peak of daily reported COVID-19 cases in the UK. A control period of 1 March to 30 April 2019 was used for comparison. Of patients presenting via the PPCI pathway during the study period, we included those with (1) an ECG consistent with STEMI ${ }^{4}$; and (2) a culprit infarct-related lesion on coronary angiography requiring intervention. Patients who did not present via the PPCI pathway, such as those self-presenting to hospital or those developing STEMI as an inpatient were not included. Patients who underwent coronary angiography revealing unobstructed coronary vessels and/or those who were given an alternative diagnosis were excluded. Data were collected from the local British Cardiac Intervention Society (BCIS) databases. We conducted two distinct analyses, first comparing data of the 2020 study period with a 2019 control group, and second within the 2020 cohort comparing patients with confirmed COVID-19 to non-COVID-19 patients.

\section{BCIS-National Institute for Cardiovascular Outcomes Research Database}

The BCIS-National Institute for Cardiovascular Outcomes Research Database collects data from all hospitals performing PCI in UK. ${ }^{12}$ Data are collected prospectively at each hospital, electronically encrypted and transferred online to a central database. Patients' survival data are obtained by linkage of patients' National Health Service numbers to the Office of National Statistics.

\section{COVID-19 status}

COVID-19 positive status was defined as either (1) the presence of a positive oro/nasopharyngeal throat swab for SARS-CoV-2 by reverse-transcriptase PCR; or (2) a clinical diagnosis based on a combination of typical symptoms, radiographic appearances ${ }^{13}$ and characteristic blood test parameters, as per the European Centre for Disease Prevention and Control criteria. ${ }^{4}$ Those who did not meet these criteria were deemed COVID-19 negative.

\section{PPCI pathway and procedural characteristics}

PPCI pathway timings and procedural characteristics were recorded in all procedural PPCI reports, as part of the UK National Cardiac Audit Programme. ${ }^{15}$ We included four time points: (1) symptom onset (pain time); (2) first call to emergency services for medical assistance (call time); (3) arrival at PPCI centre (door time); (4) first coronary intervention restoring perfusion to infarct-related artery (IRA, balloon time). Total ischaemic time was defined as the period from symptoms onset to balloon time.

Procedural characteristics of interest included: vessel(s) attempted; total number of vessels attempted $(\mathrm{n})$; total number of lesions attempted (n); total number of stents inserted $(\mathrm{n})$; total length of stent used $(\mathrm{mm})$; widest diameter balloon used $(\mathrm{mm})$; use of glycoprotein IIb/IIIa inhibitor; use of aspiration thrombectomy and 'thrombolysis in myocardial infarction' (TIMI) flow grade $^{16}$ in the IRA at the end of the case.

Presentations following out-of-hospital cardiac arrest or with cardiogenic shock were identified. Cardiogenic shock was defined in the BCIS registry as persistent hypotension with clinical evidence of hypoperfusion (cool, clammy, oliguric, altered mental status) with dependence on inotropes or mechanical left ventricular support to correct this situation. ${ }^{17}$ 


\section{Clinical characteristics and outcomes}

All-cause mortality during STEMI-related hospitalisation, admission to an intensive care unit (ICU) and total length of inpatient stay (days) were determined from electronic patient records and discharge summaries. In addition, baseline demographic characteristics and admission blood tests including high-sensitivity troponin-T, creatinine, ferritin, haemoglobin, $\mathrm{C}$ reactive protein (CRP) and lymphocyte count were also retrieved.

\section{Patient/public involvement statement}

There was no involvement of patients and the public in this study

\section{Statistical analysis}

Data analysis was performed using GraphPad Prism V.7.00 (GraphPad Software, La Jolla, California, USA). Continuous data were presented as a mean \pm SD or median (IQR) and compared using Student's t test (parametric) or Mann-Whitney U (non-parametric). Normality was assessed using the Kolmogorv-Smirnov test. Categorical data were presented as numbers with percentages and compared using Pearson's $\chi^{2}$ test and OR (95\% CI). A p value of $<0.05$ was deemed to be of statistical significance.

\section{RESULTS}

A total of 788 patients fulfilled the inclusion criteria (348 during the 2020 study period and 440 patients during the 2019 control period). There was a $21 \%$ decrease in STEMI presentations in 2020 (incident rate ratio 0.79). Four patients in the 2020 study period received upfront systemic thrombolysis as the initial reperfusion strategy but still required bailout PPCI. No patient in the 2019 control period received systemic thrombolysis. Within the 2020 group, 46 patients (13.2\%) fulfilled the criteria of COVID-19 infection and 302 were COVID-19 negative.

\section{Effect of the pandemic on clinical pathways: 2020 versus 2019 cohort}

No significant difference was observed between the 2020 and 2019 cohorts with regards to baseline demographics (table 1) and procedural characteristics (table 2). Aspiration thrombectomy and rates of cases completed with TIMI flow less than 3 were similar between both groups (respectively, $19.5 \%$ (68) vs $20.9 \%$ (92), $\mathrm{p}=0.64$, and $9.5 \%$ (33) vs $7.1 \%$ (31), $\mathrm{p}=0.21$ ). There was no significant difference in pain to first call for help (82 (30-360) $\min$ in 2020 vs 90 (22-269) $\min$ in 2019, $\mathrm{p}=0.58)$ or door to balloon (48 (34-65) min in 2020 vs $48(35-70) \mathrm{min}$

Table 1 Comparison of ST-segment elevation myocardial infarction (STEMI) admissions: 2020 study cohort versus 2019 control cohort: demographic and primary percutaneous coronary intervention (PPCl) pathway characteristics

\begin{tabular}{|c|c|c|c|c|}
\hline & \multirow{2}{*}{$\begin{array}{l}2020 \text { study cohort } \\
(\mathrm{n}=348)\end{array}$} & \multirow{2}{*}{$\begin{array}{l}2019 \text { control cohort } \\
(n=440)\end{array}$} & \multirow[b]{2}{*}{$P$ value } & \multirow[b]{2}{*}{ OR $(95 \% \mathrm{Cl})$} \\
\hline & & & & \\
\hline \multicolumn{5}{|l|}{ Baseline demographic characteristics } \\
\hline Age (years) & $63(55-71)$ & $63(55-73)$ & 0.36 & \\
\hline Male sex & $278(80 \%)$ & $343(78 \%)$ & 0.51 & \\
\hline Diabetes & $86(24.7 \%)$ & $106(24.1 \%)$ & 0.87 & \\
\hline Hypertension & $178(51.2 \%)$ & $217(49.3 \%)$ & 0.61 & \\
\hline Hyperlipidaemia & $124(35.6 \%)$ & $154(35 \%)$ & 0.85 & \\
\hline Smoking & $145(41.6 \%)$ & $245(55.7 \%)$ & $<0.001^{*}$ & \\
\hline Previous myocardial infarction & $43(12.4 \%)$ & $60(13.6 \%)$ & 0.61 & \\
\hline Stroke & $11(3.2 \%)$ & $18(4.1 \%)$ & 0.53 & \\
\hline Previous $\mathrm{PCl}$ & $43(12.4 \%)$ & $63(14.3 \%)$ & 0.42 & \\
\hline Previous CABG & $4(1.2 \%)$ & $12(2.7 \%)$ & 0.12 & \\
\hline Peripheral vascular disease & $10(2.9 \%)$ & $8(1.8 \%)$ & 0.32 & \\
\hline Renal disease & $12(3.5 \%)$ & $13(3 \%)$ & 0.69 & \\
\hline Family history of IHD & $41(11.8 \%)$ & $65(14.8 \%)$ & 0.22 & \\
\hline \multicolumn{5}{|l|}{ PPCI pathway characteristics } \\
\hline Pain—First call (min) & $82(30-360)$ & $90(22-269)$ & 0.58 & \\
\hline First call—Door (min) & $87(62-118)$ & 75 (57-95) & $0.001^{*}$ & \\
\hline Door—Balloon (min) & $48(34-65)$ & $48(35-70)$ & 0.35 & \\
\hline Total ischaemic time (min) & $282(173-618)$ & $246(157-536)$ & $0.049^{*}$ & \\
\hline Out of hospital cardiac arrest & $10.8 \%(38)$ & $8.9 \%(39)$ & 0.36 & 1.24 (0.78 to 1.98$)$ \\
\hline
\end{tabular}

Baseline demographic characteristics and PPCl pathway timings of patients with STEMI admitted during the study periods in 2020 versus 2019.

${ }^{*}$ Denotes statistical significance $(p<0.05)$. 
Table 2 Comparison of ST-segment elevation myocardial infarction (STEMI) admissions: 2020 study cohort versus 2019 control cohort: procedural characteristics and clinical endpoints

\begin{tabular}{|c|c|c|c|c|}
\hline & 2020 study cohort & 2019 control cohort & \multirow[b]{2}{*}{$P$ value } & \multirow[b]{2}{*}{ OR $(95 \% \mathrm{Cl})$} \\
\hline & $(n=348)$ & $(n=440)$ & & \\
\hline \multicolumn{5}{|l|}{ Procedural characteristics } \\
\hline Lesions treated & $1(1-1)$ & $1(1-1)$ & 0.15 & \\
\hline Vessels treated & $1(1-1)$ & $1(1-1)$ & 0.81 & \\
\hline Stents & $1(1-2)$ & $1(1-2)$ & 0.57 & \\
\hline Total length of stent $(\mathrm{mm})$ & $30(21-38)$ & $28(20-38)$ & 0.38 & \\
\hline Widest balloon (mm) & $3.5(3-4)$ & $3.5(3-4)$ & 0.56 & \\
\hline Cardiogenic shock & $47(13.5 \%)$ & $55(12.5 \%)$ & 0.68 & 1.09 (0.72 to 1.64$)$ \\
\hline Gp2b3a inhibitor use & $142(41 \%)$ & $159(36 \%)$ & 0.16 & 1.23 (0.92 to 1.63$)$ \\
\hline Thrombus aspiration use & $68(19.5 \%)$ & $92(20.9 \%)$ & 0.64 & 0.91 (0.65 to 1.30$)$ \\
\hline TIMI flow $<3$ at end of case & $33(9.5 \%)$ & $31(7.1 \%)$ & 0.21 & 1.32 (0.82 to 2.34$)$ \\
\hline \multicolumn{5}{|l|}{ Clinical endpoints } \\
\hline ICU admission & $37(10.6 \%)$ & $43(9.8 \%)$ & 0.69 & 1.10 (0.69 to 1.73$)$ \\
\hline Length of stay (days) & $3(2-4)$ & $3(2-5)$ & $<0.001^{*}$ & \\
\hline In-hospital mortality & $38(10.9 \%)$ & $8.6 \%(38)$ & 0.28 & 1.30 (0.81 to 2.08 ) \\
\hline
\end{tabular}

Procedural characteristics and clinical endpoints of patients with STEMI admitted during the study periods in 2020 versus 2019.

*Denotes statistical significance $(p<0.05)$.

ICU, intensive care unit; TIMI, thrombolysis in myocardial infarction.

in 2019, $\mathrm{p}=0.35$ ). First call to door time was significantly longer in the 2020 cohort compared with the 2019 cohort (87 (62-118) min vs 75 (57-95) min, $\mathrm{p}<0.001$; figure 1 ). There was no significant difference in ICU admission (10.6\% (37) in 2020 vs $9.8 \%$ (43) in $2019, \mathrm{p}=0.69$ ) or in-hospital all-cause mortality $(10.9 \%$ (38) in $2020 \mathrm{vs}$ $8.6 \%$ (38) in 2019, $\mathrm{p}=0.28$; table 2).

\section{Clinical presentation and outcomes in 2020: COVID-19 positive versus COVID-19 negative}

There was no significant difference in baseline characteristics between the COVID-19 positive and COVID-19 negative cohorts (table 3 ) with the exception of greater hyperlipidaemia in the COVID-19 positive group (52.2\%
(24) vs $33.1 \%$ (100), $\mathrm{p}=0.012$ ) and more frequent history of previous PCI in the COVID-19 negative group (2.2\% (1) vs $13.3 \%$ (40), $\mathrm{p}=0.03$ ). As expected, there was a statistically significant difference with respect to lower lymphocyte count, elevated ferritin and CRP in the COVID-19 positive group. No difference was seen with respect to high sensitivity Troponin $\mathrm{T}$ between the two groups (table 2).

With regards to pathway timings (figure 1), there were no statistically significant differences in median time from pain to first call (103 (30-410) min vs 80 (30-331) min, $\mathrm{p}=0.44)$ or first call to door $(88(68-144)$ min vs 86 (60-114) min, $\mathrm{p}=0.21)$. Door to balloon time was Comparison of PPCl pathway timings

i) Pain to first call time

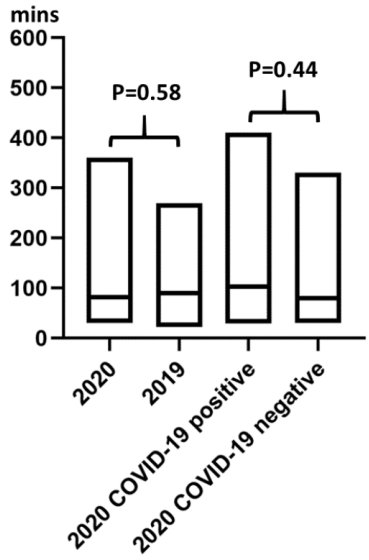

ii) First call to door time

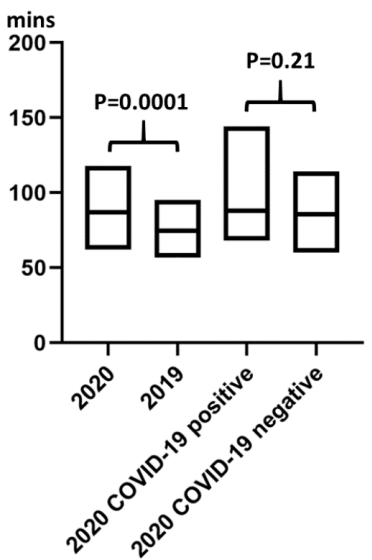

iii) Door to balloon time

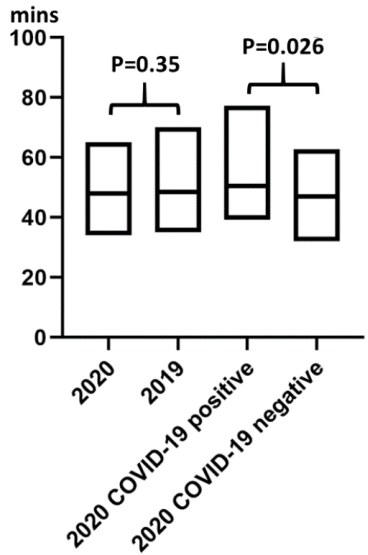

Figure 1 Pathway data are displayed as an IQR (box plot) and median (bold horizontal line within the box plot). 
Table 3 Comparison of COVID-19 positive ST-segment elevation myocardial infarction (STEMI) versus COVID-19 negative STEMI: baseline demographic characteristics, primary percutaneous coronary intervention (PPCI) pathway characteristics and admission blood tests

\begin{tabular}{|c|c|c|c|c|}
\hline & $\begin{array}{l}\text { CovID-19 positive } \\
(n=46)\end{array}$ & $\begin{array}{l}\text { COVID-19 negative } \\
(n=302)\end{array}$ & $P$ value & OR $(95 \% \mathrm{Cl})$ \\
\hline \multicolumn{5}{|l|}{ Baseline demographic characteristics } \\
\hline Age (years) & $63(58-67)$ & $63(55-72)$ & 0.66 & \\
\hline Male sex & $37(80.4 \%)$ & $241(79.8 \%)$ & 0.66 & \\
\hline Diabetes & $15(32.6 \%)$ & $71(23.5 \%)$ & 0.18 & \\
\hline Hypertension & $25(54 \%)$ & $153(50.7 \%)$ & 0.64 & \\
\hline Hyperlipidaemia & $24(52.2 \%)$ & $100(33.1 \%)$ & $0.012^{*}$ & \\
\hline Smoking & $19(41.3 \%)$ & $126(41.7 \%)$ & 0.96 & \\
\hline Previous myocardial infarction & $5(10.9 \%)$ & $38(12.6 \%)$ & 0.74 & \\
\hline Stroke & $1(2.2 \%)$ & $10(3.3 \%)$ & 0.68 & \\
\hline Previous $\mathrm{PCl}$ & $1(2.2 \%)$ & $40(13.3 \%)$ & $0.03^{*}$ & \\
\hline Previous CABG & $0(0 \%)$ & $4(1.3 \%)$ & 0.43 & \\
\hline Peripheral vascular disease & $2(4.4 \%)$ & $8(2.7 \%)$ & 0.52 & \\
\hline Renal disease & $1(2.2 \%)$ & $10(3.3 \%)$ & 0.68 & \\
\hline Family history of IHD & $7(15.2 \%)$ & $34(11.3 \%)$ & 0.44 & \\
\hline \multicolumn{5}{|l|}{ Admission blood tests } \\
\hline Haemoglobin (g/L) & $137(116-151)$ & $141(129-152)$ & 0.15 & \\
\hline Lymphocytes (×10\%/L) & $1.3(0.88-1.7)$ & $1.6(1.2-2.2)$ & $0.003^{*}$ & \\
\hline Aspartate aminotransferase (IU/L) & $66(33-217)$ & $86(30-231)$ & 0.74 & \\
\hline Alanine transaminase $(\mathrm{IU} / \mathrm{L})$ & $46(32-65)$ & $32(22-59)$ & 0.087 & \\
\hline Ferritin $(\mu \mathrm{g} / \mathrm{L})$ & $427(213-1529)$ & $176(93-313)$ & $<0.001^{*}$ & \\
\hline C reactive protein (mg/L) & $28(3-122)$ & $5(3-23)$ & $0.002^{*}$ & \\
\hline Creatinine $(\mu \mathrm{mol} / \mathrm{L})$ & $83(68-96)$ & $81(68-98)$ & 0.66 & \\
\hline High-sensitivity troponin $\mathrm{T}$ (ng/L) & $749(174-2617)$ & $419(83-2260)$ & 0.18 & \\
\hline \multicolumn{5}{|l|}{ PPCl pathway characteristics } \\
\hline Pain—First call (min) & $103(30-410)$ & $80(30-331)$ & 0.44 & \\
\hline First call—Door (min) & $88(68-144)$ & $86(60-114)$ & 0.21 & \\
\hline Door-Balloon (min) & $51(39-77)$ & $47(32-63)$ & $0.026^{*}$ & \\
\hline Total ischaemic time (min) & $360(223-1418)$ & $257(172-580)$ & $0.008^{*}$ & \\
\hline Out of hospital cardiac arrest & $5(10.9 \%)$ & $33(10.9 \%)$ & 0.99 & $0.99(0.40$ to 2.69$)$ \\
\hline
\end{tabular}

Baseline demographic characteristics, admission blood tests, PPCI pathway timings of patients with STEMI and concurrent COVID-19 and those without COVID-19 admitted during the study periods in 2020.

CABG, coronary artery bypass grafting; IHD, ischaemic heart disease.

significantly increased for COVID-19 positive patients (51 (39-77) min vs 47 (32-63) min, $\mathrm{p}=0.026)$.

Within recorded procedural characteristics (table 4), in the COVID-19 positive cohort the use of aspiration thrombectomy $(30.4 \%$ (14) vs $17.9 \%$ (54), OR 2.01 (95\% CI 0.99 to 4.05 ), $\mathrm{p}=0.046$ ) and glycoprotein $\mathrm{IIb} /$ IIIa inhibitor use $(56.5 \%$ (26) vs $38.7 \%$ (117), OR 2.06 (95\% CI 1.12 to 3.87 ), $\mathrm{p}=0.022$ ) was significantly higher. COVID-19 positive patients had significantly higher rates of procedures with a final TIMI flow less than 3 (19.6\% (9) vs $8 \%$ (24), OR 2.82 (95\% CI 1.16 to 6.45$)$, $\mathrm{p}=0.012)$. The total length of stent used was higher in the COVID-19 positive group (38 $\mathrm{mm}(24-48)$ vs $28 \mathrm{~mm}$ (20-38), $\mathrm{p}=0.012$ ). In addition, COVID-19 positive patients with STEMI had higher rates of postprocedural
ICU admission (32.6\% (15) vs 9.3\% (28) OR 5.74 (95\% CI 2.24 to 9.89$), \mathrm{p}<0.001)$, in-hospital all-cause mortality (21.7\% (10) vs $9.3 \%$ (28) OR 2.72 (95\% CI 1.25 to 5.82 ), $\mathrm{p}=0.012)$ and longer in-hospital length of stay (4 (3-9) days vs 3 (2-4) days, $\mathrm{p}<0.001)$.

\section{DISCUSSION}

The global COVID-19 pandemic has resulted in significant disruption to healthcare systems. In particular, there is a growing body of evidence worldwide demonstrating a significant decrease of up to $40 \%$ in the volume of patients presenting through PPCI services during the pandemic. ${ }^{5}{ }^{18-20}$ Our data support these findings with heart attack services in London experiencing a $21 \%$ 
Table 4 Comparison of COVID-19 positive ST-segment elevation myocardial infarction (STEMI) versus COVID-19 negative STEMI: procedural characteristics and clinical endpoints

\begin{tabular}{|c|c|c|c|c|}
\hline & $\begin{array}{l}\text { COVID-19 positive } \\
(n=46)\end{array}$ & $\begin{array}{l}\text { COVID-19 negative } \\
(n=302)\end{array}$ & $P$ value & OR (95\% Cl) \\
\hline \multicolumn{5}{|l|}{ Procedural characteristics } \\
\hline Lesions treated & $1(1-1)$ & $1(1-1)$ & 0.44 & \\
\hline Vessels treated & $1(1-1)$ & $1(1-1)$ & 0.53 & \\
\hline Stents & $1(1-2)$ & $1(1-2)$ & 0.99 & \\
\hline Total length of stent (mm) & 38 (24-48) & $28(20-38)$ & $0.012^{\star}$ & \\
\hline Widest balloon (mm) & $3.5(2.5-3.75)$ & $3.5(3-4)$ & 0.14 & \\
\hline Cardiogenic shock & $6(13 \%)$ & $41(13.6 \%)$ & 0.92 & 0.95 (0.40 to 2.30$)$ \\
\hline Gp2b3a inhibitor use & $26(56.5 \%)$ & $117(38.7 \%)$ & $0.022^{*}$ & 2.06 (1.12 to 3.87$)$ \\
\hline Thrombus aspiration use & $14(30.4 \%)$ & $54(17.9 \%)$ & $0.046^{*}$ & 2.01 (0.99 to 4.05$)$ \\
\hline TIMI flow $<3$ at end of case & $9(19.6 \%)$ & $24(8 \%)$ & $0.012^{\star}$ & 2.82 (1.16 to 6.45$)$ \\
\hline \multicolumn{5}{|l|}{ Clinical endpoints } \\
\hline ICU admission & $15(32.6 \%)$ & $28(9.3 \%)$ & $<0.001^{*}$ & 5.74 (2.24 to 9.89$)$ \\
\hline Length of stay (days) & $4(3-9)$ & $3(2-4)$ & $<0.001^{*}$ & \\
\hline In-hospital mortality & $10(21.7 \%)$ & $28(9.3 \%)$ & $0.012^{\star}$ & 2.72 (1.25 to 5.82$)$ \\
\hline
\end{tabular}

Procedural characteristics and clinical endpoints of patients with STEMI and concurrent COVID-19 and those without COVID-19 admitted during the study periods in 2020.

ICU, intensive care unit; TIMI, thrombolysis in myocardial infarction.

decrease in cases compared with the same period in 2019. There could be a number of explanations for this observation. First, patients experiencing chest pain may have been reluctant to seek medical attention either in fear of viral transmission or not wishing to burden the already stretched healthcare system. Second, patients with no cardiac history may have attributed their myocardial infarction symptoms to COVID-19 infection and, as per government advice at the time, did not seek immediate help. Finally, those patients who did seek help may not have been prioritised by the ambulance service who were under immense pressure at the time and a proportion of these patients may have deteriorated and died before receiving medical attention. In addition to the fall in acute presentations, survey data suggest a corresponding increase in the incidence of cardiac related complications associated with untreated STEMI ${ }^{21}$; however, the long-term implications remain unclear.

The key to an effective PPCI service is minimisation of total ischaemic time. ${ }^{4}$ Critical to this pathway is prompt identification of STEMI, rapid transfer to a PPCI centre and immediate revascularisation on arrival. Delays at any stage during this pathway may increase myocardial ischaemia time resulting in reduced myocardial salvage, larger infarct size and subsequent long term morbidity. ${ }^{23}$ COVID-19 resulted in multiple potential delays to this pathway. Our analysis comparing the 2020 study population to the 2019 control population in a busy metropolitan city importantly revealed comparable door to balloon times between the two groups, contrary to previous reports ${ }^{24}$ with comparable in-hospital mortality rates. This suggests that modifications to the existing in-hospital PPCI pathways, such as routine use of PPE and redeployment of staff to other clinical areas, neither delayed the time taken to achieve coronary revascularisation (door to balloon time) or resulted in worse outcomes. However, the median ischaemic time was 36 min longer in 2020 than 2019, driven predominantly by an increase in the median time from first call for help to arrival at a PPCI centre. The likely explanation for this is the impact of a high volume of COVID-19-illness related calls overwhelming paramedic emergency services leading to a delayed response time, although additional time for paramedics to don PPE may also have contributed. The increased door to balloon time and subsequent increased ischaemic time for COVID-19 positive patients compared with COVID-19 negative patients in 2020 likely reflects the increased procedural complexity of these cases rather than system-related delays as all centres mandated full PPE for all PPCI cases given the unknown COVID-19 status at time of procedure. While systemic thrombolysis has been suggested as an alternative strategy to managing patients with STEMI during the pandemic, ${ }^{3}$ our data suggest that even at the peak of COVID-19 related admissions to hospital, it was possible to maintain effective PPCI services.

Current literature suggest that patients with COVID-19 presenting with STEMI have higher rates of in-hospital mortality, compared with those without concurrent COVID$19{ }^{25}$ Our data support this finding with more than doubling in the rate of in-hospital mortality for these patients. Furthermore, the increased frequency of ICU admission and longer length of stay have significant implications on hospital resources. Importantly, there was no significant difference in the baseline characteristics between COVID-19 positive and COVID-19 negative patients. The cause of this 
excess mortality is not clear but it is likely that a number of factors may be contributory. First, COVID-19 itself is associated with high in-hospital mortality and the infarct in addition to systemic illness results in poor prognosis particularly in those with significant comorbidity. ${ }^{5}$ Second, delays in presentation result in longer ischaemic which is known to be associated with larger infarct size and therefore these patients are more likely to develop fatal post-MI complications. ${ }^{26}$ Third, patients with COVID-19 appear to be highly prothrombotic compared with COVID-19 negative patients, with studies demonstrating high incidence of pulmonary thromboembolic disease as well as thrombi in other organs such at the kidneys. ${ }^{8}$ High coronary thrombus burden at presentation is independently associated with increased rates of major adverse cardiovascular events and mortality. ${ }^{27}{ }^{28}$ Furthermore, intraprocedural distal embolisation of thrombus may disrupt microvascular function resulting in angiographic no reflow and increase infarct size. ${ }^{29}$ Thrombus burden determination is highly subjective and as such we employed thrombus aspiration usage and reduced TIMI flow at the end of the procedure as surrogates. Reduced TIMI flow is frequently seen with distal embolisation of thrombotic material. ${ }^{30}$ This study demonstrates an increased use of thrombus aspiration and glycoprotein IIb/IIIa inhibitors within the COVID-19 cohort, with higher rates of reduced TIMI flow, suggesting a higher thrombotic burden when compared with non-COVID-19 patients with STEMI. Glycoprotein IIb/IIIa inhibitors have a class IIa indication for adjunctive therapy with PCI with evidence of high thrombotic burden ${ }^{4}$ and may be of benefit in patients with COVID-19 although with increased risk of bleeding. A targeted antithrombotic approach may be of benefit in these patients to decrease rates of distal embolisation of thrombus and subsequently decrease infarct size in order to improve long-term outcomes but further prospective studies are required.

Choudry et $a t^{6}$ have recently published a single centre experience demonstrating a signal of increased thrombus burden in COVID-19 patients with STEMI ( $\mathrm{n}=39$ ) with higher use of aspiration thrombectomy and glycoprotein IIb/IIIa inhibitors, as well as higher postintervention TIMI thrombus grade and lower resultant myocardial blush score. Importantly, our multicentre study includes the largest cohort of COVID-19 positive patients with STEMI $(n=46)$ compared with COVID-19 negative patients with similar presentation during the same time period, and in addition to confirming these findings also demonstrates a significant increase in mortality in the COVID-19 positive group and delays in door to balloon time. Our additional control group of patients with STEMI from 2019 provides key insights into the effects of modifications to existing PPCI pathways. Mafham et $a t^{20}$ have highlighted a substantial reduction in acute coronary syndrome presentations in England during the COVID-19 pandemic period compared with 2019. Our study provides further analysis based on the presence or absence of concurrent COVID-19 infection and validates their findings by providing details of both the heart attack pathways and procedural characteristics.
Limitations of the current study include its retrospective design in the first instance and therefore the potential introduction of information bias. Additionally, we only included patients receiving PCI for STEMI and data on those subjects with STEMI who did not undergo PCI (including those receiving systemic thrombolysis only and those managed medically in the first instance) were not included in our analysis. Moreover, patients who underwent coronary angiography that revealed no culprit lesion were excluded from the analysis. It has been reported that in COVID-19 positive patients presenting with STEMI between 33\% and 39\% of cases demonstrate no culprit lesion on angiography. ${ }^{525}$ This represents a large proportion of admissions through the PPCI pathway and the effects of pathway modifications on this cohort within our study are not assessed. While this is a large study of PPCI services in a metropolitan city, the COVID-19 positive group was relatively small making up less than $5 \%$ of the cohort and therefore findings within this group should be interpreted with caution. Moreover, data are limited to in-hospital outcomes. Long-term prospective follow-up will be required to determine the true association between COVID-19 and excess mortality following STEMI. Furthermore, the threshold used by emergency service to initiate the PPCI pathway, during the pandemic, may have been influenced by factors relating to infection control and resource management. This may have contributed to the reduction seen in PPCI activations worldwide in 2020. Finally, the outcome of those patients who did not present to the PPCI service is unknown. They may have significantly worse late outcomes, with heart failure, arrhythmia and death as yet unmeasured in the community.

\section{CONCLUSION}

The COVID-19 pandemic has resulted in unprecedented strain on hospital services in London and as a consequence of this, a number of modifications to the existing PPCI pathway were required. While these factors resulted in increased ambulance response times, there was no adverse effect on door-to-balloon times or mortality. This suggests that even under the extreme pressures of increased COVID-19 related ambulance call-outs, delayed response times, routine PPE use and increased procedural complexity, well-established clinical pathways can be maintained without compromising patient outcomes. Second, patients with COVID-19 infection presenting with STEMI exhibit higher thrombotic burden and are at significant increased risk of death.

\footnotetext{
Author affiliations

${ }^{1}$ Department of Cardiology, Royal Free London NHS Foundation Trust, London, United Kingdom

${ }^{2}$ Institute of Cardiovascular Science, University College London, London, United Kingdom

${ }^{3}$ Department of Cardiology, Imperial College Healthcare NHS Trust, London, United Kingdom

${ }^{4}$ Department of Cardiology, Barts Health NHS Trust, London, United Kingdom ${ }^{5}$ Department of Cardiology, Royal Brompton \& Harefield NHS Foundation Trust, London, United Kingdom
} 
${ }^{6}$ Department of Cardiology, St George's University Hospitals NHS Foundation Trust, London, United Kingdom

${ }^{7}$ Department of Cardiology, King's College Hospital NHS Foundation Trust, London, United Kingdom

${ }^{8}$ Department of Cardiology, Guy's and St Thomas' NHS Foundation Trust, London, United Kingdom

Twitter Callum D Little @callumlittle6 and Ozan M Demir @DrOzanDemir

Acknowledgements The authors would like to acknowledge the following individuals who provided valuable contributions to the study: N Patel, SS Kalra, D Patel, T Lockie, G Coghlan, E Smith, G Manmathan, M Curtis and S Cashin.

Collaborators Niket Patel, Sundeep S Kalra, Deven Patel, Tim Lockie, Gerry Coghlan, Elliot Smith, Gavin Manmathan, M Curtis and S Cashin.

Contributors CDL, TK, LC and RR cowrote the paper. RJJ, GBC, AA, MC, RK, OMD, $\mathrm{LOL}$ and BW contributed to data collection at participating hospitals. SF, JCS, $\mathrm{DP}, \mathrm{PM}, \mathrm{MD}, \mathrm{AJ}, \mathrm{SJW}$ and IM provided oversight and quality control of data from participating hospitals. All authors approved the final manuscript.

Funding This work was supported by the National Institute for Health Research University College London Hospitals Biomedical Research Centre. CDL and RDR gratefully acknowledge funding from the Wellcome/EPSRC Centre for Interventional and Surgical Sciences (WEISS)(203145Z/16/Z).

Competing interests None declared.

Patient consent for publication Not required.

Ethics approval All patient identifiable information was removed before database merging and analysis. The audit was registered with the hospital audit departments at each centre. Because this analysis was performed on anonymised data from mandatory audit, the local research ethics committee advised that formal ethical approval was not required.

Provenance and peer review Not commissioned; externally peer reviewed.

Data availability statement Deidentified participant data are available upon reasonable request. Please contact Callum D Little at callumlittle@nhs.net

Open access This is an open access article distributed in accordance with the Creative Commons Attribution 4.0 Unported (CC BY 4.0) license, which permits others to copy, redistribute, remix, transform and build upon this work for any purpose, provided the original work is properly cited, a link to the licence is given, and indication of whether changes were made. See: https://creativecommons.org/ licenses/by/4.0/.

\section{ORCID iDs}

Callum D Little http://orcid.org/0000-0002-2267-0924

Ritesh Kanyal http://orcid.org/0000-0003-4079-2578

Philip MacCarthy http://orcid.org/0000-0002-5963-0109

\section{REFERENCES}

1 WHO. Situation report 38: coronavirus disease (COVID-19), 2020. Available: https://www.who.int/docs/default-source/coronaviruse/ situation-reports/20200227-sitrep-38-covid-19.pdf?sfvrsn= 9f98940c 6

2 Medicine C for EB. COVID 19: admissions to hospital, 2020. Available: https://www.cebm.net/covid-19/covid-19-uk-hospitaladmissions/

3 Mahmud E, Dauerman HL, Welt FGP, et al. Management of acute myocardial infarction during the COVID-19 pandemic: a consensus statement from the Society for cardiovascular angiography and interventions (Scal), the American College of cardiology (ACC), and the American College of emergency physicians (ACEP). Catheter Cardiovasc Interv 2020;96:336-45.

4 Ibanez B, James S, Agewall S, et al. 2017 ESC guidelines for the management of acute myocardial infarction in patients presenting with ST-segment elevation. Eur Heart J 2018;39:119-77.

5 Stefanini GG, Montorfano M, Trabattoni D, et al. St-Elevation myocardial infarction in patients with COVID-19: clinical and angiographic outcomes. Circulation 2020;141:1-7.

6 Choudry FA, Hamshere SM, Rathod KS, et al. High Thrombus Burden in Patients With COVID-19 Presenting With ST-Segment Elevation Myocardial Infarction. J Am Coll Cardiol 2020;76:1168-76.
7 Inciardi RM, Adamo M, Lupi L, et al. Characteristics and outcomes of patients hospitalized for COVID-19 and cardiac disease in northern Italy. Eur Heart J 2020;41:1821-9.

8 Connors JM, Levy JH. COVID-19 and its implications for thrombosis and anticoagulation. Blood 2020;135:2033-40.

9 Varga Z, Flammer AJ, Steiger P, et al. Endothelial cell infection and endotheliitis in COVID-19. The Lancet 2020;395:1417-8.

10 Lopes da Silva R. Viral-Associated thrombotic microangiopathies. Hematol Oncol Stem Cell Ther 2011;4:51-9.

11 Helms J, Tacquard C, Severac F, et al. High risk of thrombosis in patients with severe SARS-CoV-2 infection: a multicenter prospective cohort study. Intensive Care Med 2020;46:1089-98.

12 Rashid M, Ludman PF, Mamas MA. British cardiovascular intervention Society registry framework: a quality improvement initiative on behalf of the National Institute of cardiovascular outcomes research (NICOR). Eur Heart J Qual Care Clin Outcomes 2019;5:292-7.

13 Long $\mathrm{C}, \mathrm{Xu} \mathrm{H}$, Shen $\mathrm{Q}$, et al. Diagnosis of the coronavirus disease (COVID-19): rRT-PCR or CT? Eur J Radiol 2020;126:108961.

14 Case definition for coronavirus disease 2019, 2020. Available: https://www.ecdc.europa.eu/en/covid-19/surveillance/casedefinition

15 Wilkinson C, Weston C, Timmis A, et al. The myocardial ischaemia national audit project (MINAP). Eur Hear $J$ - Qual Care Clin Outcomes 2020.

16 Ganz W. The thrombolysis in myocardial infarction (TIMI) trial. N Engl J Med 1985;313:1018.

17 Kunadian V, Qiu W, Ludman P, et al. Outcomes in patients with cardiogenic shock following percutaneous coronary intervention in the contemporary era: an analysis from the BCIS database (British cardiovascular intervention Society). JACC Cardiovasc Interv 2014;7:1374-85.

18 Garcia S, Albaghdadi MS, Meraj PM, et al. Reduction in STsegment elevation cardiac catheterization laboratory activations in the United States during COVID-19 pandemic. J Am Coll Cardiol 2020;75:2871-2.

19 De Filippo O, D'Ascenzo F, Angelini F, et al. Reduced rate of hospital admissions for ACS during Covid-19 outbreak in northern Italy. $N$ Engl J Med 2020;383:88-9.

20 Mafham MM, Spata E, Goldacre R, et al. COVID-19 pandemic and admission rates for and management of acute coronary syndromes in England. Lancet 2020;396:1-9.

21 Pessoa-Amorim G, Camm CF, Gajendragadkar P, et al. Admission of patients with STEMI since the outbreak of the COVID-19 pandemic: a survey by the European Society of cardiology. Eur Heart J Qual Care Clin Outcomes 2020;6:210-6.

22 De Luca G, Suryapranata H, Zijlstra F, et al. Symptom-onsetto-balloon time and mortality in patients with acute myocardial infarction treated by primary angioplasty. J Am Coll Cardiol 2003;42:991-7.

23 Stone GW, Selker HP, Thiele $\mathrm{H}$, et al. Relationship between infarct size and outcomes following primary $\mathrm{PCl}$ : patient-level analysis from 10 randomized trials. J Am Coll Cardiol 2016;67:1674-83.

24 Tam C-CF, Cheung K-S, Lam S, et al. Impact of coronavirus disease 2019 (COVID-19) outbreak on outcome of myocardial infarction in Hong Kong, China. Catheter Cardiovasc Interv 2020. doi:10.1002/ ccd.28943. [Epub ahead of print: 05 May 2020].

25 Bangalore S, Sharma A, Slotwiner A, et al. St-Segment elevation in patients with Covid-19 - a case series. N Engl J Med Overseas Ed 2020;382:2478-80.

26 Scholz KH, Maier SKG, Maier LS, et al. Impact of treatment delay on mortality in ST-segment elevation myocardial infarction (STEMI) patients presenting with and without haemodynamic instability: results from the German prospective, multicentre FITT-STEMI trial. Eur Heart J 2018;39:1065-74.

27 Sianos G, Papafaklis MI, Daemen J, et al. Angiographic stent thrombosis after routine use of drug-eluting stents in ST-segment elevation myocardial infarction: the importance of thrombus burden. J Am Coll Cardiol 2007;50:573-83.

28 Singh $\mathrm{M}$, Berger $\mathrm{PB}$, Ting $\mathrm{HH}$, et al. Influence of coronary thrombus on outcome of percutaneous coronary angioplasty in the current era (the Mayo clinic experience). Am J Cardiol 2001;88:1091-6.

29 Fokkema ML, Vlaar PJ, Svilaas T, et al. Incidence and clinical consequences of distal embolization on the coronary angiogram after percutaneous coronary intervention for ST-elevation myocardial infarction. Eur Heart J 2009;30:908-15.

30 Henriques JPS, Zijlstra F, Ottervanger JP, et al. Incidence and clinical significance of distal embolization during primary angioplasty for acute myocardial infarction. Eur Heart J 2002;23:1112-7. 